\title{
Role of Melatonin in Preterm Birth
}

\author{
Zeeshan Ahmad Khan', Gopinath Mondal' , Chandresh Sharma², \\ Shahkar Falak ${ }^{3}$, Abuzar Ansari ${ }^{4}$, and Asamanja Chattoraj ${ }^{5}$ \\ 'Biological Rhythm Laboratory, Institute of Bioresources and Sustainable Development, Imphal, India \\ ${ }^{2}$ Translational Health Science and Technology Institute, NCR Biotech Science Cluster, Faridabad, India \\ ${ }^{3}$ Department of Biotechnology, Techno India University, Kolkata, India \\ ${ }^{4}$ Department of Zoology, Aligarh Muslim University, Aligarh, India \\ ${ }^{5}$ Department of Animal Science, Kazi Nazrul University, Asansol, India
}

\begin{abstract}
Preterm babies are at major risk and it is the second-largest direct cause of child deaths in children aging less than 5 years. Melatonin, a naturally synthesized hormone secreted by the pineal gland, offers antioxidant, anti-inflammatory, immunomodulatory, anti-apoptotic, and antibacterial activity. As preterm is multifactorial, several researchers have studied the role of melatonin in reducing the risk of preterm birth. In this review, the authors tried to study the role of melatonin in preterm birth. After a brief background of preterm birth and melatonin biosynthesis, we will discuss the role of melatonin in pregnancy. Furthermore, the role of melatonin in various preterm associated diseases such as respiratory and neural diseases will be addressed.
\end{abstract}

Key Words: Melatonin; Preterm birth; Circadian rhythm disorders; Pregnancy; Circadian rhythm

Received: October 31, 2020 Revised: November 24, 2020 Accepted: November 26, 2020

Corresponding author: Zeeshan Ahmad Khan, M.Tech, PhD, Biological Rhythm Laboratory, Institute of Bioresources and Sustainable Development, Takyelpat, Imphal, India.

Tel: 91-873-1867720,E-mail: acezeeshan@live.com

Corresponding author: Asamanja Chattoraj, MSc, PhD, Department of Animal Science, Kazi Nazrul University, Paschim Brdhaman, West Bengal 713340, Asansol, India.

Tel: 91-341-225-2024, Fax: 91-341-227-1024, E-mail: asamanja.chattoraj@gmail.com

@ This is an Open Access article distributed under the terms of the Creative Commons Attribution Non-Commercial License (https://creativecommons.org/licenses/bync/4.0) which permits unrestricted non-commercial use, distribution, and reproduction in any medium, provided the original work is properly cited.

\section{INTRODUCTION}

Globally, preterm birth is the leading cause of neonatal death and child deaths $<5$ years [1]. In 2015, preterm birth alone responsible for $35.5 \%$ of newborn deaths and $17.8 \%$ of death age under 5 years [2]. The burden of preterm birth is highly significant in developing countries (18\%) than the developed nations (5\%) [1]. Exceptionally, the United States is one of the top 10 countries with the highest proportion of preterm births [1]. Although recent development in neonatal care has significantly improved, prematurely born babies still have a higher risk for long-term morbidities, such as neurodevelopmental disorder, cognitive impairments, etc. [3,4]. Therefore, the development of preventive measures for preterm birth; and novel treatment strategy for premature infants are urgently needed.

Melatonin (5-methoxy- $\mathrm{N}$-acetyltryptamine) is a multifunctional hormone known for its role in numerous psychological and physiological responses including biological rhythms, endocrine regulation, antioxidant activity, sleep maintenance, and immune responses [5-10]. A successful pregnancy requires coordination from all the physiological parameters between mother and fetus. Alteration of these crucial features may result in preterm births [11-16]. Evidence suggests that maternal melatonin level is essential for fetal development and differential level of serum melatonin in pregnant and non-pregnant women has been reported (Figure 1) [17,18]. Okatani et al. [19] reported that melatonin able to cross the placental barrier, indicating maternal melatonin responsible for influencing the internal rhythms of the offspring. Disruption of internal melatonin rhythms may lead to preterm birth [20]. Most recently, one study concluded that night-shift working and continuous exposure to artificial light at unconventional times might compromise the success of full-term birth and the health of newborn infants [21]. Conversely, melatonin supplementation during pregnancy significantly increased gestation 


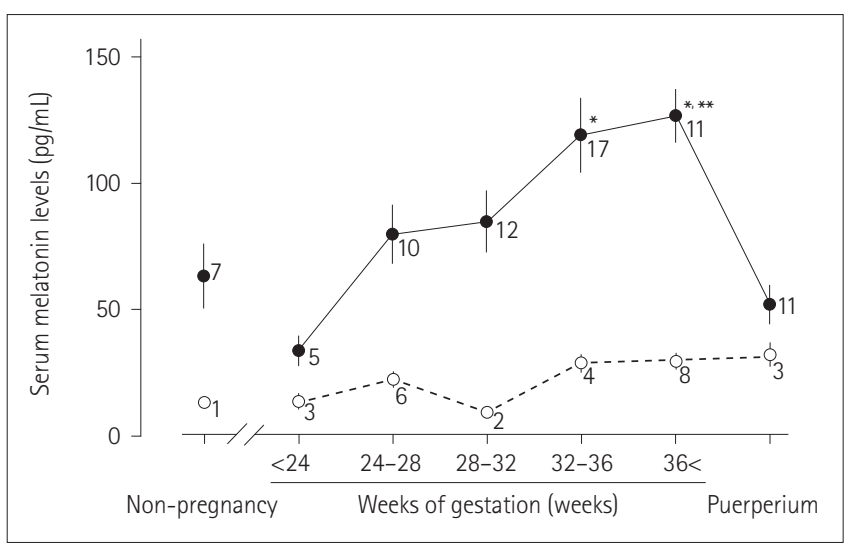

Figure 1. Changes of maternal serum melatonin levels at nighttime (solid line) or daytime (dotted line) in the normal singleton pregnancy. Values are means and SEM for the number of given patients indicated beside each dot. Data at daytime less than lower limit (5.6 $\mathrm{pg} / \mathrm{mL}$ ) were excluded from the analysis. ${ }^{*} \mathrm{p}<0.01$ compared with the non-pregnancy values, <24-week values, or puerperium values. ${ }^{* *} p<0.05$ compared with the 24-28-week value. Reprinted from Nakamura et al. J Pineal Res 2001;30:29-33, with permission of John Wiley and Sons [18].

length and birth weight [22]. Furthermore, during pregnancy, melatonin treatment decreases preterm birth and perinatal brain injury [23]. Besides, melatonin intervention can also reduce brain damage in preterm neonates [24].

Melatonin had already shown its therapeutic potential for sleep disorders, jet lag, or shift work $[25,26]$. Generally, the action of melatonin is determined by the nocturnal secretion pattern. For instance, melatonin is involved in the regulation of sleep in diurnal species, which is associated with the length of night, or several metabolic processes associated with energy balance and seasonal control of photoperiodic functions. The external changes in the photoperiod can directly influence the metabolism in various species of vertebrates [6,27]. The artificial light at night and skyglow have been demonstrated to disturb the biological rhythm by influencing the secretion of melatonin which may disturb the pregnancy $[18,27,28]$. In this review, firstly, we explored the preclinical and clinical evidence to extend our knowledge on the role of melatonin during pregnancy. It is hypothesized that melatonin supplementation may improve the developmental and cognitive disorders in preterm infants. Therefore, secondly, we discuss the recent progress regarding the favorable effects of exogenous melatonin on preterm birth, with particular emphasis on respiratory diseases and brain injury. Finally, we discussed how chronodisruption is associated with a high risk of preterm birth.

\section{MELATONIN BIOSYNTHESIS}

Melatonin can be considered as ancient as it seems to have appeared around 2.5-3.0 billion years ago in photosynthetic cyanobacteria [29]. Moreover, melatonin has a highly conserved pattern of biosynthesis. It is high during night and nadir at day time; therefore, it is considered the most important "chronobiotic" molecule and endogenous neurohormonal signal of darkness $[5,10,30]$. The chronobiotic molecule melatonin, an indoleamine, is mostly synthesized in the pineal gland of vertebrates. Information from studies of the last few years has enriched us about the presence and synthesis of melatonin in the retina, gut, ovary, and brain [31-35]. The synthesis of melatonin is very much conserved and classically involves four enzymatic processes using the amino acid tryptophan as the initial substrate. 5-hydroxytryptophan is produced from tryptophan by tryptophan hydroxylase. The serotonin or 5-hydroxytryptamine (5-HT) is the next product by the action of 5-hydroxytryptophan decarboxylase. The 5-HT is acetylated to $\mathrm{N}$-acetylserotonin by arylalkylamine $\mathrm{N}$-acetyltransferase. Finally, methylation of the last product by hydroxyindole-O-methyltransferase forms the melatonin [36-39].

Local melatonin synthesis has been reported in other tissues and cells in mammals, which has a minor effect on plasma or cerebrospinal fluid (CSF) levels of melatonin. For instance, melatonin is synthesized in the retina in a circadian manner and participates in the regulation of retinal physiology, including retinal light sensitivity during the night [40]. The gastrointestinal epithelium also produces melatonin from its precursor serotonin, which is thought to come from enterochromaffin cells. In the gut, melatonin seems to participate in the regulation of intestinal motility, immune system responses, ion transportation in the lower gut, and the release of peptides involved in energy balance such as peptide YY [41].

\section{FUNCTION OF MELATONIN IN PREGNANCY}

Melatonin decreases in conditions associated with serious outcomes for the fetus and seems to be involved in preeclampsia and intrauterine growth restriction [11]. Melatonin treatment during human normal or abnormal pregnancy has been studied for a large range of conditions and at different times during the gestational period. Considering the ethical issues, it is more difficult to study a normally occurring pregnancy, than an in vitro fertilization (IVF) one. Melatonin administration started before IVF-cycles, continued during pregnancy, and was associated with improved pregnancy outcomes [8]. Melatonin receptors are widespread in the human fetus from early fetal development. Also, it appears that the fetuses' sleep patterns develop in late pregnancy, melatonin being the regulating factor. A normal sleep pattern is involved in neurodevelopment and there is solid evidence that melatonin is involved in fetal neuroprotection. Thus, the influence of melatonin on the developing human fetus may not be limited to entertaining the circadian rhythmicity. Melatonin research showed that it plays an important role in pregnancy and parturition. The passage of maternal melatonin through the placenta exposes the fetus to a daily melatonin rhythm of low concentrations during the day and high concentrations at night. Therefore, first, melatonin is involved in inducing a circadian manner of functioning in fetal organs.

The ability of melatonin to promote embryo development in 
different species has been reported. When mouse embryos were cultured in a medium containing melatonin, increased blastocyst development rates were observed [17]. Melatonin has a beneficial role in the in vitro development of rodent embryos found in the 2-cell stage [19] and helped the maturation of bovine blastocysts [20]. Suppression of maternal plasma melatonin circadian rhythm by continuous light exposure during the second half of gestation showed several effects on fetal development. First, it induced intrauterine growth retardation. Second, in the fetal adrenal in vivo it markedly affected the mRNA expression level of the clock genes and clock-controlled genes, as well as lowered the content and modified the rhythm of corticosterone. Third, an altered in vitro fetal adrenal response to adrenocorticotropic hormone (ACTH) of both, corticosterone production and relative expression of clock genes and steroidogenic genes was observed. All these changes were reversed when the mother received a daily dose of melatonin during the subjective night [21].

Torres-Farfan et al. [22] reported that maternal melatonin decreased cortisol production in the fetal adrenal gland of the capuchin monkey. In another study on sheep, they found that melatonin had direct inhibitory effects on the noradrenalin-stimulated fetal cerebral artery contraction, the release of glycerol by brown adipose tissue, and on ACTH-induced secretion of cortisol by the fetal adrenal gland. Low levels or a lack of a circadian rhythm of the fetal corticosterone may be the cause of the intrauterine growth retardation that has been previously reported. The lack of maternal melatonin (induced by pinealectomy) during the early stages of gestation was found to disrupt the drinking behavior of rat pups, an effect reversed by the administration of exogenous melatonin to the dam [23]. Melatonin is important in normal placental development and function, a function supported by the placenta melatonin receptors expression during early pregnancy [24]. Whereas melatonin synthesis is classically considered to take place in the cytoplasm, in the past 5 years, non-rhythmic melatonin synthesis has been reported to occur in isolated mitochondria from mouse brain cells and oocytes, as well as in plant mitochondria and chloroplasts. The full meaning of this intriguing finding remains to be clarified, including the question of the effect of mitochondrial melatonin synthesis in comparison to pineal melatonin synthesis.

\section{ROLE OF MELATONIN IN PRETERM BIRTH}

It is hypothesized that deprivation of long-term endogenous melatonin levels may link with neurological and developmental disabilities in premature neonates. In fact, after full-term birth, the neonate unable to generate melatonin for the first 8-16 weeks-a phase is called transient melatonin deficiency [1-5]. Although previous studies showed that the suprachiasmatic nucleus and the pineal gland had appeared to mature in early fetal life [3], however, the neurological circuitry that controls these structures remain immature [6,7]. Therefore, in both preterm and full-term neo- nates (absence of maternal melatonin), the uprising of the circadian pattern is crucially dependent on the neurodevelopmental status rather than the home environment [8]. Prematurity itself is negatively associated with the maturation of the neurological network, which controls melatonin secretion [9]. Evidence suggests that the impairment for the development of the neurological network delayed the onset of pineal melatonin secretion [2,5]. Consequently, in the preterm neonates, the deficiency of endogenous melatonin levels is significantly longer than the full-term neonate $[1,2,4,5]$. Extensive research has shown that abnormal brain, lungs, and respiratory system development most frequently occur in the premature infant. Data from the previous study suggest that this may be associated with a prolonged deficiency of pineal melatonin levels, which may be restored with melatonin supplementation [10]. In this section, we discuss the recent progress regarding the beneficial effects of melatonin on preterm birth, with particular emphasis on respiratory diseases and brain injury.

\section{The pivotal role of melatonin in respiratory diseases}

Bronchopulmonary dysplasia (BPD), a chronic lung disease that requires respiratory support at birth, is most frequently appeared in preterm infants whose gestation age $<29$ weeks [11]. Pathogenesis of BPD has been attributed to multiple factors, including prematurity of lung tissue, superabundant of the inflammatory state, and disruption of the repairing process following injury [12-14]. In light of the scientific advances in the field of neonatology, newer and effective perinatal strategies have come out to fight against BPD, for instance, new formulations of surfactant (makes breathing easier), application of corticosteroids, and non-invasive ventilation [15]. Despite this, BPD remains one of the most common complications associated with prematurity [16]. Most recently, one randomized clinical trial had conducted on 100 premature neonates who had been suffering from severe respiratory distress syndrome (RDS) by mechanical ventilation [17]. In this study, the premature neonates were allocated into two groups: group one population received melatonin supplementation and group two without melatonin supplementation. The results of this study revealed that the melatonin treated group significantly declined in the length of incubator stay of neonates and markedly decreased in the development of established cases of BPD, in comparison to that of premature neonates who did not take melatonin [17], indicating melatonin may use as an adjuvant therapy for the prevention of BPD in premature neonates. In another clinical trial, it has been reported that the inflammatory markers, including interleukin-6 (IL-6), interleukin-8 (IL-8), and tumor necrosis factor- $\alpha$ (TNF- $\alpha$ ), were significantly higher at one day, three days, and at seven days in preterm neonates with RDS, whereas melatonin treatment can block these inflammatory components and likely reduce the severity of RDS in preterm newborns [18]. Furthermore, melatonin treatment in preterm infants with RDS not only decreased inflammatory markers but also reduced nitrite/nitrate levels in comparison with the placebo-treated group [19]. In BPD, immature lung recruits inflammatory cells 
and overexpress inflammatory molecules, which are considered the mainstem pathologic pathway to tissue damage [20]. Based on pathological consideration, melatonin may exert beneficial effects in the incidence of BPD, particularly in preterm newborns. However, the data from the aforementioned studies cannot support the beneficial effects of melatonin for long-term respiratory outcomes in preterm infants, which need further investigation. In hyperoxia-induced BPD animals, the lung myeloperoxidase and nitrite/nitrate ratio were decreased through melatonin intervention [21]. Moreover, melatonin treated group significantly increased antioxidant enzymatic activities, including glutathione peroxidase, superoxide dismutase, and catalase activity [42]. These findings indicate melatonin contributes to lung architecture development and protects lung during the critical processes of alveolarization following preterm birth.

\section{Neuroprotective role of melatonin in brain injury}

Recent developments in neonatology have focused on fetal brain injury, which is considered one of the leading causes of morbidity and mortality in preterm infants [22]. Intraventricular hemorrhage (IVH, where CSF is produced) and white matter abnormalities frequently appear in preterm infants, resulting in neurosensory and cognitive disabilities [23-25]. The cellular and molecular mechanisms of preterm infants' brain injury to be complex and multifactorial. Evidence suggests that oxidative stress is among the most important risk factors for brain damage in preterm newborns [24]. Previous findings suggest that neuronal cells are more vulnerable to hypoxic-ischemic injury in the preterm brain than in term neonates [24], particularly related to high levels of reactive oxygen species that are responsible for significant neuronal damage [43]. Relatively the immature brains are more prone to oxidative stress than the matured brain because the immature brain contains a higher proportion of blood vessels, and their cortex poorly developed when compared with mature ones [44]. Particularly in preterm neonates, morbidity and mortality are significantly influenced by their ability to maintain physiological homeostasis [45]. Interestingly, disruption of the antioxidant defense system most often appeared in preterm infants, consequently, they are unable to counteract the detrimental effects of free radicals [45]. Data from several preclinical studies suggest that melatonin exerts neuroprotective effects in the preterm infant brain. For example, in the umbilical cord occlusion induced asphyxia, the oxidative stress was decreased with melatonin treatment, in both preterm and near-term fetal sheep [22]. Chowdhury and Maitra [32] also reported that melatonin reduced cell death in the fetal brain following intrauterine asphyxia by decreasing inflammation in preterm fetal sheep.

One recent study demonstrated that the administration of melatonin before and after hypoxia in immature rats has markedly attenuated brain injury and improved behavioral asymmetry, suggesting melatonin would be a potentially safe drug to perinatal brain damage in humans [46]. To better understand the difference between pharmacokinetic parameters of melatonin in pre- term infants and adults, Merchant et al. [34] had compared the preterm infant pharmacokinetic profile (PP) with extrapolating adult pharmacokinetic data. The principal findings of this study demonstrated that the PP of melatonin in preterm neonates differs from that of adults. Therefore, for preterm infants, the melatonin dosage cannot be extrapolated from adult studies. These results are similar to those reported by Carloni et al. [35]. Most recently, in preterm fetal sheep, Yawno et al. [24] examined whether melatonin could recover white matter brain development when administered after an acute hypoxic-ischemic insult. It is apparent from this study that hypoxic-ischemia causes a notable increase in apoptosis, microglial activation, and oxidative stress in white matter areas. Whereas melatonin treatment significantly reduced apoptosis, inflammation, and oxidative stress following hypoxic-ischemia [36].

\section{PRETERM BIRTH IN SHIFT WORKERS AND INSOMNIA}

Chronodisruption leads to reproductive dysfunction and a low level of melatonin appears to be a key contributor to preterm birth and associated diseases that develop in adult life (the concept of fetal programming) (Figure 2) [47]. Women working an evening shift or a night shift, or who are trans meridian traveling, report an increased or decreased menstrual cycle length, changes in the duration and amount of menstrual bleeding, and dysmenorrhea [48-50]. These symptoms are not subjective, as they correlate with changes in the hormonal profile of ovarian and pituitary origin, such as a high follicular stimulating hormone (FSH) and prolonged follicular stage of the ovarian cycle [50]. The working environment also affects pregnancy outcomes. The shift work while pregnant is also associated with a high risk of prematurity and/ or low for gestational age babies, spontaneous abortion, and subfecundity [51,52]. Shift work and jet lag effects on health may be

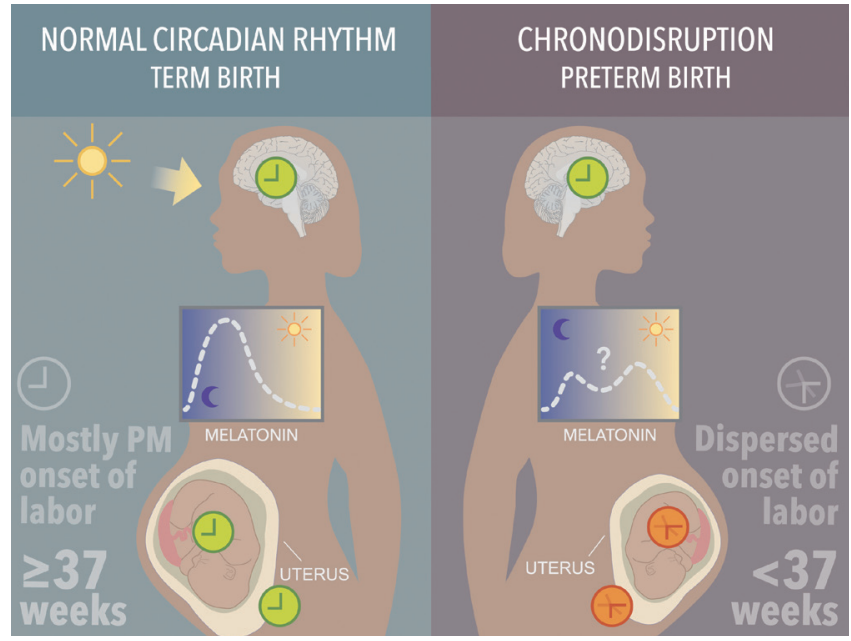

Figure 2. Chronodisruption may be a risk factor for preterm birth. Chronodisruption may cause a misalignment in the central and peripheral circadian system, altered melatonin secretion patterns, which induce poor reproductive outcomes. Reprinted from Reschke et al. Best Pract Res Clin Obstet Gynaecol 2018;52:60-67, with permission of Elsevier [47]. 
explained by the secondary reduction of the total amount of sleep. FSH concentration in women who sleep less than $8 \mathrm{~h} /$ night lowers by $1 / 5$ compared to women with longer sleep duration [53]. Total or partial sleep deprivation increases luteinizing hormone (LH) amplitude, estradiol, and FSH concentrations in normal cycling women, and increased estrogen is associated with a high risk of breast cancer. Cumulatively, shift work, or poor sleep may become a crucial factor in preterm birth.

\section{THERAPEUTIC POTENTIAL OF MELATONIN IN PRETERM BIRTH}

Preterm birth (birth before 37-week gestation) nowadays is a serious health issue worldwide. Over 13 million babies were born in the preterm condition in 2005 and more than 1 million infants dying every year due to preterm born. Those who survived face an increased risk of morbidities, such as cerebral palsy, autism, schizophrenia, blindness, and hearing loss [54]. In recent days many advances have been made in the medical field of obstetrics and neonatology, but the rate of premature delivery has not decreased yet over the past 20 to 30 years. To date, there is no efficient therapy to prevent preterm labor and increase the survival of offspring as no precise pathways have been discovered for this preterm parturition. Despite that, from some epidemiological and experimental data, it is revealed that maternal infection and inflammation are the key factors in preterm birth. Approximately $40 \%$ of all premature deliveries occur to mothers who have an intrauterine infection [55]. Working outside of regular work, like shift working, night working highly prevalent in both industrialized and post-industrialized societies. Particularly health care professionals, more are women and many of them are of reproductive age which can have a hazardous effect on pregnancy and fetal development [52]. The circadian rhythm aligns many physiological and behavioral rhythms in the mother according to that fetus also synchronizes its oscillating circadian rhythms and this tightly coordinated mechanism helps in the developmental processes [56,57]. During night work circadian rhythm of the mother's body is disrupted due to the exposure to light [58]. This type of repeated and consecutive changes in maternal circadian rhythm affects fetal development as fetuses failed to synchronize their oscillating circadian rhythms with mother. Exposure to light during the dark period suppresses the amplitude of melatonin secretion, which might further affect the circadian rhythms of the mother body. Melatonin possess antioxidant, a free radical scavenger and anti-inflammatory properties which indicate it have a role in reducing oxidative stress [59-61]. It is evident that the melatonin is elevated during pregnancy and it can cross the placenta, blood-brain barrier of fetus without showing any risk for mother and fetus [62,63]. Bacterial infection in the uterus, placenta, and fetal tissues stimulates cytokine and chemokine responses by leukocytes and other cells which leads to the release of prostaglandins (PGs), nitric oxide (NO), and matrix metalloproteinases [55]. It has been reported that PGs, NO, and TNF- $\alpha$ are mainly responsible for preterm labor in several rodent models as well as in humans [64-66]. A high level of plasma melatonin during late pregnancy helps in gestation maintenance by stimulating progesterone production and inhibiting PG synthesis and uterine contractility [67]. In mice, melatonin protects from lipopolysaccharide (LPS)-induced fetal death, intrauterine growth restriction, and cellular stress in the placenta [68] while other research demonstrated that maternally administered melatonin differentially regulates LPS-induced pro-inflammatory and antiinflammatory cytokine levels in maternal serum, amniotic fluid, fetal liver, and fetal brain [69].

It has been reported that increased NO, PG, and cytokine plays a significant role in preterm delivery. Whatever, meloxicam (a COX-2 inhibitor), aminoguanidine (an iNOS inhibitor), etanercept (a competitive inhibitor of TNF- $\alpha$ ) prevent inflammationinduced preterm delivery $[65,70]$, besides that the impairment of PG/COX-2 system, decrease in NO levels, diminishing cytokine production or combination of these treatments may be a therapeutic strategy to prevent preterm labor [67]. In that way melatonin could be a promising resource in the managing of preterm labor as it decreases PGs, NO, IL-1 $\beta$, IL-6, COX-2, and TNF- $a$ levels and can regulate the circadian rhythm in mice uterus and other mammals $[71,72]$. Besides that, melatonin is a very safe compound for human use, may be considered as a novel tocolytic agent to treatment against inflammation-associated preterm birth in clinical trials [23].

\section{CONCLUSIONS}

In conclusion, it may be claimed that melatonin may play a pivotal role in reducing preterm birth. While the clinical applicability of melatonin is yet to be elucidated, it remains a very promising candidate in avoiding preterm birth or the diseases associated with preterm birth. More clinical studies, pharmacokinetics, and pharmacodynamic analysis of melatonin in pregnant women, placenta, and the embryo are required to further confirm the role and potency of melatonin in reducing preterm birth.

Acknowledgments

None

\section{Conflicts of Interest}

The authors have no potential conflicts of interest to disclose.

\section{Author Contributions}

Conceptualization: Zeeshan Ahmad Khan, Gopinath Mondal. Data curation: Zeeshan Ahmad Khan, Gopinath Mondal, Asamanja Chattoraj, Chandresh Sharma, Abuzar Ansari, Shahkar Falak. Project administration: Zeeshan Ahmad Khan, Gopinath Mondal, Abuzar Ansari, Chandresh Sharma, Shahkar Falak. Resources: Zeeshan Ahmad Khan, Gopinath Mondal, Asamanja Chattoraj, Chandresh Sharma, Shahkar Falak. Supervision: Zee- 
shan Ahmad Khan, Abuzar Ansari, Asamanja Chattoraj, Chandresh Sharma, Shahkar Falak. Writing_original draft: Zeeshan Ahmad Khan, Gopinath Mondal, Abuzar Ansari, Asamanja Chattoraj, Chandresh Sharma, Shahkar Falak. Writing-review \& editing: Zeeshan Ahmad Khan, Gopinath Mondal, Asamanja Chattoraj, Chandresh Sharma, Shahkar Falak.

\section{ORCID iDs}

\section{Zeeshan Ahmad Khan (1)}

https://orcid.org/0000-0002-0737-0253

Gopinath Mondal (1)

https://orcid.org/0000-0002-9771-0767

Asamanja Chattoraj (1)

https://orcid.org/0000-0002-8656-7459

\section{REFERENCES}

1. Blencowe H, Cousens S, Oestergaard MZ, Chou D, Moller AB, Narwal R, et al. National, regional, and worldwide estimates of preterm birth rates in the year 2010 with time trends since 1990 for selected countries: a systematic analysis and implications. Lancet 2012;379:2162-2172.

2. Liu L, Oza S, Hogan D, Chu Y, Perin J, Zhu J, et al. Global, regional, and national causes of under-5 mortality in 2000-15: an updated systematic analysis with implications for the Sustainable Development Goals. Lancet 2016; 388:3027-3035.

3. Mwaniki MK, Atieno M, Lawn JE, Newton CR. Long-term neurodevelopmental outcomes after intrauterine and neonatal insults: a systematic review. Lancet 2012;379:445-452.

4. Spittle A, Orton J, Anderson P, Boyd R, Doyle LW. Early developmental intervention programmes post-hospital discharge to prevent motor and cognitive impairments in preterm infants. Cochrane Database Syst Rev 2012; 12:CD005495.

5. Masters A, Pandi-Perumal SR, Seixas A, Girardin JL, McFarlane SI. Melatonin, the hormone of darkness: from sleep promotion to Ebola treatment. Brain Disord Ther 2014;4:1000151.

6. Cipolla-Neto J, Amaral FG, Afeche SC, Tan DX, Reiter RJ. Melatonin, energy metabolism, and obesity: a review. J Pineal Res 2014;56:371-381.

7. Li Y, Li S, Zhou Y, Meng X, Zhang JJ, Xu DP, et al. Melatonin for the prevention and treatment of cancer. Oncotarget 2017;8:39896-39921.

8. Tarocco A, Caroccia N, Morciano G, Wieckowski MR, Ancora G, Garani G, et al. Melatonin as a master regulator of cell death and inflammation: molecular mechanisms and clinical implications for newborn care. Cell Death Dis 2019;10:317.

9. Fan LL, Sun GP, Wei W, Wang ZG, Ge L, Fu WZ, et al. Melatonin and doxorubicin synergistically induce cell apoptosis in human hepatoma cell lines. World J Gastroenterol 2010;16:1473-1481.

10. Pévet P. Melatonin. Dialogues Clin Neurosci 2002;4:57-72.

11. van den Berg CB, Chaves I, Herzog EM, Willemsen SP, van der Horst GTJ, Steegers-Theunissen RPM. Early- and late-onset preeclampsia and the DNA methylation of circadian clock and clock-controlled genes in placental and newborn tissues. Chronobiol Int 2017;34:921-932.

12. Loy SL, Cheung YB, Cai S, Colega MT, Godfrey KM, Chong YS, et al. Maternal night-time eating and sleep duration in relation to length of gestation and preterm birth. Clin Nutr 2020;39:1935-1942.

13. Hsu JY, James KE, Bormann CL, Donahoe PK, Pépin D, Sabatini ME. Müllerian-inhibiting substance/anti-Müllerian hormone as a predictor of preterm birth in polycystic ovary syndrome. J Clin Endocrinol Metab 2018;103: 4187-4196.

14. Moore TA, Ahmad IM, Zimmerman MC. Oxidative stress and preterm birth: an integrative review. Biol Res Nurs 2018;20:497-512.

15. Okun ML, Schetter CD, Glynn LM. Poor sleep quality is associated with preterm birth. Sleep 2011;34:1493-1498.

16. Sampah MES, Hackam DJ. Dysregulated mucosal immunity and associated pathogeneses in preterm neonates. Front Immunol 2020;11:899.

17. Voiculescu SE, Zygouropoulos N, Zahiu CD, Zagrean AM. Role of melato- nin in embryo fetal development. J Med Life 2014;7:488-492.

18. Nakamura Y, Tamura H, Kashida S, Takayama H, Yamagata Y, Karube A, et al. Changes of serum melatonin level and its relationship to feto-placental unit during pregnancy. J Pineal Res 2001;30:29-33.

19. Okatani Y, Okamoto K, Hayashi K, Wakatsuki A, Tamura S, Sagara Y. Maternal-fetal transfer of melatonin in pregnant women near term. J Pineal Res 1998;25:129-134.

20. McCarthy R, Jungheim ES, Fay JC, Bates K, Herzog ED, England SK. Riding the rhythm of melatonin through pregnancy to deliver on time. Front Endocrinol (Lausanne) 2019;10:616.

21. Nehme PA, Amaral FG, Middleton B, Lowden A, Marqueze E, França-Junior I, et al. Melatonin profiles during the third trimester of pregnancy and health status in the offspring among day and night workers: a case series. Neurobiol Sleep Circadian Rhythms 2019;6:70-76.

22. Torres-Farfan C, Valenzuela FJ, Mondaca M, Valenzuela GJ, Krause B, Herrera EA, et al. Evidence of a role for melatonin in fetal sheep physiology: direct actions of melatonin on fetal cerebral artery, brown adipose tissue and adrenal gland. J Physiol 2008;586:4017-4027.

23. Lee JY, Song H, Dash O, Park M, Shin NE, McLane MW, et al. Administration of melatonin for prevention of preterm birth and fetal brain injury associated with premature birth in a mouse model. Am J Reprod Immunol 2019;82:e13151.

24. Yawno T, Mahen M, Li J, Fahey MC, Jenkin G, Miller SL. The beneficial effects of melatonin administration following hypoxia-ischemia in preterm fetal sheep. Front Cell Neurosci 2017;11:296.

25. Park EJ, Park YM. Melatonin and sleep problems in children with autism spectrum disorder. Chronobiol Med 2020;2:47-51.

26. Cho $\mathrm{CH}$, Lee Y. The chronobiologic-based practical approach to shift work. Chronobiol Med 2019;1:103-106.

27. Khan ZA, Labala RK, Yumnamcha T, Devi SD, Mondal G, Sanjita Devi H, et al. Artificial Light at Night (ALAN), an alarm to ovarian physiology: a study of possible chronodisruption on zebrafish (Danio rerio). Sci Total Environ 2018;628-629:1407-1421.

28. Lee E, Kim M. Light and life at night as circadian rhythm disruptors. Chronobiol Med 2019;1:95-102.

29. Zheng X, Tan DX, Allan AC, Zuo B, Zhao Y, Reiter RJ, et al. Chloroplastic biosynthesis of melatonin and its involvement in protection of plants from salt stress. Sci Rep 2017;7:41236.

30. Ameen IA. Melatonin the hormone of darkness: a review. Indian J Nat Sci 2018;8:14410-14415.

31. Jimenez-Jorge S, Guerrero JM, Jimenez-Caliani AJ, Naranjo MC, Lardone PJ, Carrillo-Vico A, et al. Evidence for melatonin synthesis in the rat brain during development. J Pineal Res 2007;42:240-246.

32. Chowdhury I, Maitra SK. Melatonin time line: from discovery to therapy. In: Watson RR, editor. 2nd ed. Boca Raton: Taylor \& Francis, 2012, p. 1-60.

33. Khan ZA, Yumnamcha T, Rajiv C, Sanjita Devi H, Mondal G, Devi SD, et al. Melatonin biosynthesizing enzyme genes and clock genes in ovary and whole brain of zebrafish (Danio rerio): differential expression and a possible interplay. Gen Comp Endocrinol 2016;233:16-31.

34. Merchant NM, Azzopardi DV, Hawwa AF, McElnay JC, Middleton B, Arendt J, et al. Pharmacokinetics of melatonin in preterm infants. Br J Clin Pharmacol 2013;76:725-733.

35. Carloni S, Proietti F, Rocchi M, Longini M, Marseglia L, D'Angelo G, et al. Melatonin pharmacokinetics following oral administration in preterm neonates. Molecules 2017;22:2115.

36. Chattoraj A, Liu T, Zhang LS, Huang Z, Borjigin J. Melatonin formation in mammals: in vivo perspectives. Rev Endocr Metab Disord 2009;10:237-243.

37. Falcón J. Cellular circadian clocks in the pineal. Prog Neurobiol 1999;58: 121-162.

38. Falcón J, Besseau L, Sauzet S, Boeuf G. Melatonin effects on the hypothalamo-pituitary axis in fish. Trends Endocrinol Metab 2007;18:81-88.

39. Klein DC, Coon SL, Roseboom PH, Weller JL, Bernard M, Gastel JA, et al. The melatonin rhythm-generating enzyme: molecular regulation of serotonin N-acetyltransferase in the pineal gland. Recent Prog Horm Res 1997; 52:307-357; discussion 357-358.

40. Brennan R, Jan JE, Lyons CJ. Light, dark, and melatonin: emerging evidence for the importance of melatonin in ocular physiology. Eye (Lond) 2007;21: 901-908.

41. Aydin M, Canpolat S, Kuloğlu T, Yasar A, Colakoglu N, Kelestimur H. Ef- 
fects of pinealectomy and exogenous melatonin on ghrelin and peptide YY in gastrointestinal system and neuropeptide $Y$ in hypothalamic arcuate nucleus: immunohistochemical studies in male rats. Regul Pept 2008;146:197203.

42. Choi CW, Kim BI, Hong JS, Kim EK, Kim HS, Choi JH. Bronchopulmonary dysplasia in a rat model induced by intra-amniotic inflammation and postnatal hyperoxia: morphometric aspects. Pediatr Res 2009;65:323-327.

43. Li B, Concepcion K, Meng X, Zhang L. Brain-immune interactions in perinatal hypoxic-ischemic brain injury. Prog Neurobiol 2017;159:50-68.

44. The developing brain. In: National Research Council (US) and Institute of Medicine (US) Committee on Integrating the Science of Early Childhood Development; Shonkoff JP, Phillips DA, editors. From neurons to neighborhoods: the science of early childhood development. Washington (DC): National Academies Press (US), 2000, chapter 8. Available at: https://www. ncbi.nlm.nih.gov/books/NBK225562/.

45. Perlman J, Kjaer K. Neonatal and maternal temperature regulation during and after delivery. Anesth Analg 2016;123:168-172.

46. Carloni S, Perrone S, Buonocore G, Longini M, Proietti F, Balduini W. Melatonin protects from the long-term consequences of a neonatal hypoxicischemic brain injury in rats. J Pineal Res 2008;44:157-164.

47. Reschke L, McCarthy R, Herzog ED, Fay JC, Jungheim ES, England SK. Chronodisruption: an untimely cause of preterm birth? Best Pract Res Clin Obstet Gynaecol 2018;52:60-67.

48. Shechter A, Boivin DB. Sleep, hormones, and circadian rhythms throughout the menstrual cycle in healthy women and women with premenstrual dysphoric disorder. Int J Endocrinol 2010;2010:259345.

49. Youseflu S, Jahanian Sadatmahalleh S, Roshanzadeh G, Mottaghi A, Kazemnejad A, Moini A. Effects of endometriosis on sleep quality of women: does life style factor make a difference? BMC Womens Health 2020;20:168.

50. Mahoney MM. Shift work, jet lag, and female reproduction. Int J Endocrinol 2010;2010:813764.

51. Davari MH, Naghshineh E, Mostaghaci M, Mirmohammadi SJ, Bahaloo M, Jafari A, et al. Shift work effects and pregnancy outcome: a historical cohort study. J Family Reprod Health 2018;12:84-88.

52. Specht IO, Hammer PEC, Flachs EM, Begtrup LM, Larsen AD, Hougaard KS, et al. Night work during pregnancy and preterm birth-A large registerbased cohort study. PLoS One 2019;14:e215748.

53. de Zambotti M, Colrain IM, Baker FC. Interaction between reproductive hormones and physiological sleep in women. J Clin Endocrinol Metab 2015; 100:1426-1433.

54. Goldenberg RL, Culhane JF, Iams JD, Romero R. Epidemiology and causes of preterm birth. Lancet 2008;371:75-84.

55. Romero R, Espinoza J, Kusanovic JP, Gotsch F, Hassan S, Erez O, et al. The preterm parturition syndrome. BJOG 2006;113:17-42.

56. Mark PJ, Crew RC, Wharfe MD, Waddell BJ. Rhythmic three-part harmony: the complex interaction of maternal, placental and fetal circadian systems. J Biol Rhythms 2017:32:534-549.
57. Reppert SM, Weaver DR, Rivkees SA. Maternal communication of circadian phase to the developing mammal. Psychoneuroendocrinology 1988;13:6378

58. Scott AJ, LaDou J. Shiftwork: effects on sleep and health with recommendations for medical surveillance and screening. Occup Med 1990;5:273-299.

59. Jensen MA, Hansen ÅM, Kristiansen J, Nabe-Nielsen K, Garde AH. Changes in the diurnal rhythms of cortisol, melatonin, and testosterone after 2, 4 , and 7 consecutive night shifts in male police officers. Chronobiol Int 2016; 33:1280-1292.

60. Tamura H, Nakamura Y, Terron MP, Flores LJ, Manchester LC, Tan DX, et al. Melatonin and pregnancy in the human. Reprod Toxicol 2008;25:291303.

61. Reiter RJ, Tan DX, Korkmaz A, Rosales-Corral SA. Melatonin and stable circadian rhythms optimize maternal, placental and fetal physiology. Hum Reprod Update 2014;20:293-307.

62. Biran V, Phan Duy A, Decobert F, Bednarek N, Alberti C, Baud O. Is melatonin ready to be used in preterm infants as a neuroprotectant? Dev Med Child Neurol 2014;56:717-723.

63. Wilkinson D, Shepherd E, Wallace EM. Melatonin for women in pregnancy for neuroprotection of the fetus. Cochrane Database Syst Rev 2016;3: CD010527.

64. Burdet J, Sacerdoti F, Cella M, Franchi AM, Ibarra C. Role of TNF- $\alpha$ in the mechanisms responsible for preterm delivery induced by $\mathrm{Stx} 2$ in rats. $\mathrm{Br} J$ Pharmacol 2013;168:946-953.

65. Burdet J, Zotta E, Cella M, Franchi AM, Ibarra C. Role of nitric oxide in shiga toxin-2-induced premature delivery of dead fetuses in rats. PLoS One 2010;5:e15127.

66. Vrachnis N, Karavolos S, Iliodromiti Z, Sifakis S, Siristatidis C, Mastorakos $\mathrm{G}$, et al. Review: impact of mediators present in amniotic fluid on preterm labour. In Vivo 2012;26:799-812.

67. Domínguez Rubio AP, Sordelli MS, Salazar AI, Aisemberg J, Bariani MV, Cella M, et al. Melatonin prevents experimental preterm labor and increases offspring survival. J Pineal Res 2014;56:154-162.

68. Wang H, Li L, Zhao M, Chen YH, Zhang ZH, Zhang C, et al. Melatonin alleviates lipopolysaccharide-induced placental cellular stress response in mice. J Pineal Res 2011;50:418-426

69. Xu DX, Wang H, Ning H, Zhao L, Chen YH. Maternally administered melatonin differentially regulates lipopolysaccharide-induced proinflammatory and anti-inflammatory cytokines in maternal serum, amniotic fluid, fetal liver, and fetal brain. J Pineal Res 2007;43:74-79.

70. Cella M, Farina MG, Dominguez Rubio AP, Di Girolamo G, Ribeiro ML, Franchi AM. Dual effect of nitric oxide on uterine prostaglandin synthesis in a murine model of preterm labour. Br J Pharmacol 2010;161:844-855.

71. Bonnefont-Rousselot D, Collin F. Melatonin: action as antioxidant and potential applications in human disease and aging. Toxicology 2010;278:55-67.

72. Karaaslan C, Suzen S. Antioxidant properties of melatonin and its potential action in diseases. Curr Top Med Chem 2015;15:894-903. 